\title{
Genetic relationships among American species of Prosopis (Leguminosae) based on enzyme markers
}

\author{
Cecilia Bessega ${ }^{1,2}$, Beatriz Ofelia Saidman ${ }^{3}$ and Juan César Vilardi ${ }^{3}$ \\ Facultad de Ciencias Exactas y Naturales, Departamento de Ecología, Genética y Evolución, \\ Buenos Aires, Argentina.
}

\begin{abstract}
In the present work, isoenzyme electrophoresis was used to analyze the variability and phenetic relationships among seven American species of genus Prosopis belonging to three different sections: $P$. argentina (Monilicarpa), $P$. glandulosa, $P$. velutina, $P$. flexuosa, $P$. ruscifolia, $P$. kuntzei (Algarobia), and $P$. reptans (Strombocarpa). The genetic variability in $P$. argentina, $P$. reptans, and $P$. kuntzei was significantly lower than in the rest of the species analyzed. The species belonging to different sections are highly differentiated, but the relationships retrieved among species belonging to the section Algarobia suggested that the series of this section are not natural groups. $P$. kuntzei is as differentiated from the remaining species of Algarobia as from $P$. reptans or $P$. argentina, suggesting that this species might be included in a different section. The series within section Algarobia are not supported by the clusters retrieved in the phenogram based on isoenzymatic data. The results suggest that the two North American species ( $P$. velutina and $P$. glandulosa) would have originated in different founder events.
\end{abstract}

Key words: Prosopis, isoenzymes, genetic variability, phenetic relationships.

Received: April 26, 2004; Accepted: November 11, 2004.

\section{Introduction}

Prosopis is a primitive legume genus that includes shrubs and trees that exhibit a high economic and ecological potential in semiarid areas. They are a component of the climax community, but they also have the ability to colonize new habitats as pioneer species. Many of its species are used in numerous countries to recover arid and semiarid regions and are considered multipurpose trees because all of their biomass can be used. Pods have high carbohydrate and protein content and are used as forage and human food. These trees have wood of high quality and adapt well to silvopastoril and agroforestal production (Roig, 1993). Prosopis involves about 44 species grouped in five sections: Prosopis and Anonychium, distributed in Africa and Asia; Strombocarpa and Algarobia, distributed in North and South America; and the monotypic section Monilicarpa, restricted to Argentina.

The section Strombocarpa includes nine species clearly differentiated morphologically from each other, and

Send correspondence to Cecilia Bessega. Facultad de Ciencias Exactas y Naturales, Departamento de Ecología, Genética y Evolución, Buenos Aires, Argentina. E-mail: cecib@bg.fcen.uba.ar. 'Present Address: Instituto de Biotecnología CICVyA - CNIA - INTA - CC 25 - 1712 - Castelar, Argentina.

${ }^{2}$ Fellow of Consejo Nacional de Investigaciones Científicas y Tecnológicas (CONICET).

${ }^{3}$ Member of CONICET. interspecific hybridization is infrequent (Burkart, 1976). Isoenzymatic studies in five of them $(P$. torquata, $P$. pubescens, $P$. strombulifera, and $P$. reptans) showed low intraspecific variability and yielded many species-specific diagnostic loci (Saidman et al., 1996).

Based on morphological grounds the section Algarobia has been divided into six series (Burkart, 1976), but the relationships among species of this section are under debate. Studies based on species of the series Pallidae, Ruscifoliae, and Chilenses indicated that natural hybrids frequently occur in zones of sympatry even between species belonging to different series (Hunziker et al., 1986). However, no interserial hybrids have been recorded involving species of the series Sericanthae. The information about hybridization involving species of the two remaining series, Denudans and Humiles, is scarce.

The species of Ruscifoliae and Chilenses studied so far exhibit high levels of variability within populations, but low biochemical and genetical differentiation among species (Saidman and Vilardi, 1987, 1993; Saidman et al., 1997, 1998a,b). The relationships observed among species in isoenzymatic (Saidman and Vilardi, 1993; Bessega et al., 2000a,b) and molecular (Ramírez et al., 1999; Saidman et al., 1998a,b; Saidman et al., 2000) analyses are not consistent with the series.

Previous works (Hunziker et al., 1986; Saidman et al., 1996) indicated that the sections Algarobia and 
Strombocarpa are highly differentiated. The speciation process in these groups as well as their adaptive strategies seem to be quite different.

The Section Monilicarpa is represented by only one isolated species, $P$. argentina Burkart endemic to Argentina. According to Burkart $(1937,1976)$ its origin and affinities of either Algarobia or Strombocarpa are uncertain. Its preference for sand dunes is in marked contrast to that of the common species of section Algarobia, which prefer heavier soils, sometimes salty clay on lower plateaus and riverbanks (Burkart, 1976). There are only a few biochemical (Burghardt and Palacios, 1997) and molecular (Ramirez et al., 1999) studies on $P$. argentina and the population genetic variability has yet to be studied.

In the present work we analyzed, by means of isoenzyme electrophoresis, the variability and phenetic relationships among $P$. argentina and six species belonging to the remaining American sections of Prosopis: $P$. glandulosa (Torr) and $P$. velutina Wooton from USA, $P$. flexuosa DC, P. ruscifolia Gris., P. kuntzei Harms (Algarobia), and $P$. reptans Bentham (Strombocarpa) from Argentina. Parameters of genetic variability and differentiation were estimated in populations of these species. This information is discussed with reference to the expected relationships according to the available morphological evidence and geographical distribution.

\section{Materials and Methods}

\section{Species, populations and sampling methods}

The present work involved two populations of $P$. argentina ("algarobilla"), one of $P$. reptans ("retortuño"), one of P. kuntzei ("itín"), four of P. ruscifolia ("vinal"), two of
P. flexuosa ("algarrobo amarillo"), four of P. glandulosa ("honey mesquite") and one of $P$. velutina ("velvet mesquite") (Table 1, Figure 1).

The species and populations sampled cover a wide range of the distribution of the genus Prosopis in America (Burkart, 1976). P. argentina (sect. Monilicarpa) is endemic in Northwestern Argentina in the Andean provinces of Catamarca, La Rioja, San Juan and northern Mendoza

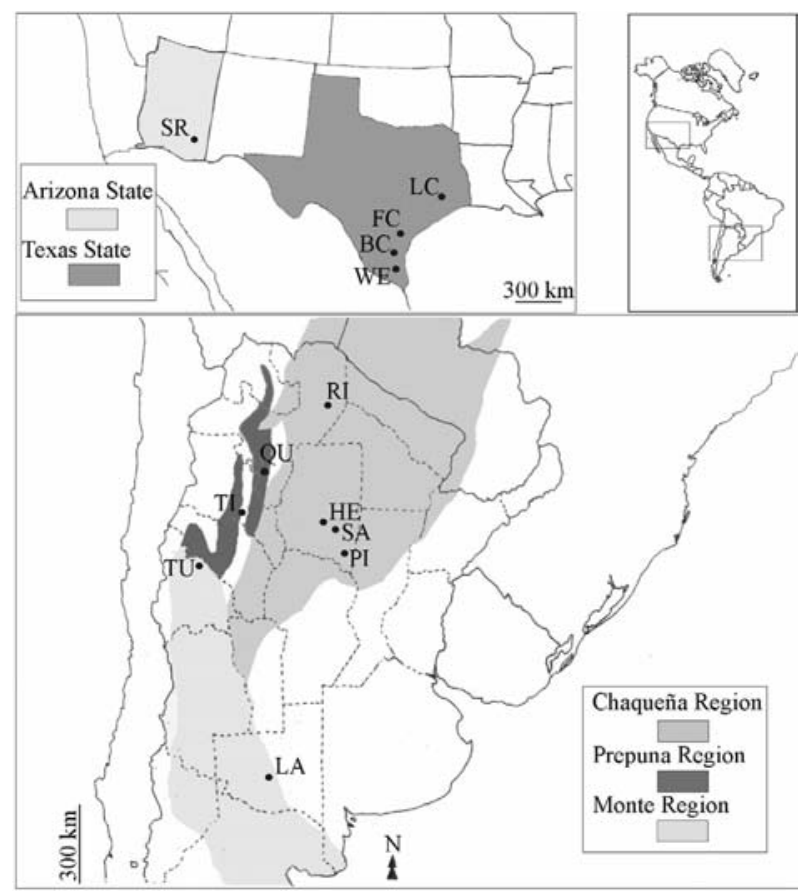

Figure 1 - Map indicating the location of the sampled populations. For references see Table 1.

Table 1 - List of the sections, series, Prosopis species and populations studied, collectors and codes used to identify populations in Figure 1.

\begin{tabular}{|c|c|c|c|c|c|}
\hline Section & Series & Species & Collection localities & Collector & Code \\
\hline \multirow[t]{2}{*}{ Monilicarpa } & & P. argentina & Tinogasta, Catamarca, Argentina & P.Villlagra $^{1}$ & TI \\
\hline & & & Tucunuco, San Juan, Argentina & P.Villlagra $^{1}$ & $\mathrm{TU}$ \\
\hline Strombocarpa & Strombocarpae & P. reptans & Herrera, S. del Estero, Argentina & BOS-JCV. ${ }^{2}$ & $\mathrm{HE}$ \\
\hline \multirow[t]{12}{*}{ Algarobia } & Sericanthae & P. kuntzei & Herrera, S. del Estero, Argentina & BOS-JCV. ${ }^{2}$ & $\mathrm{HE}$ \\
\hline & Ruscifoliae & P. ruscifolia & Herrera, S. del Estero, Argentina & BOS-JCV. ${ }^{2}$ & $\mathrm{HE}$ \\
\hline & & & Rivadavia, Salta, Argentina & BOS-JCV. ${ }^{2}$ & RI \\
\hline & & & Pinto, S. del Estero, Argentina & BOS-JCV. ${ }^{2}$ & PI \\
\hline & & & Sarmiento, S. del Estero, Argentina & BOS-JCV. ${ }^{2}$ & SA \\
\hline & Chilenses & P.flexuosa & La Amarga, La Pampa, Argentina & BOS-JCV. ${ }^{2}$ & LA \\
\hline & & & Quilmes, Tucumán, Argentina & BOS-JCV. ${ }^{2}$ & QU \\
\hline & & P. glandulosa & Weslaco, Texas, USA & J.Evans. ${ }^{3}$ & WE \\
\hline & & & La Copita, Texas, USA & J.Evans. ${ }^{3}$ & $\mathrm{LC}$ \\
\hline & & & Bell Co., Texas, USA & J.Evans. ${ }^{3}$ & $\mathrm{BC}$ \\
\hline & & & Frio Co., Texas, USA & J.Evans. ${ }^{3}$ & FC \\
\hline & & P. velutina & Santa Rita, Arizona, USA & J.Evans. ${ }^{3}$ & SR \\
\hline
\end{tabular}

${ }^{1}$ IADIZA = Instituto Argentino de Investigaciones en Zonas Áridas (Mendoza Argentina). ${ }^{2}$ UBA = Universidad de Buenos Aires (Buenos Aires, Argentina). ${ }^{3}$ GRS-USDA = Grassland Research Station USDA/ARS (USA). 
(Argentina). P. reptans var. reptans (Strombocarpa) grows in central Argentina and Peru. From the Algarobia section, $P$. glandulosa and $P$. velutina ranges involve southwestern United States and Mexico. The other three species are restricted to South America: P. flexuosa can be found in Prepuna and Monte regions (northwestern and central Argentina); P. ruscifolia occurs in the Chaqueña region (Santiago del Estero, Chaco and Formosa provinces, Argentina); and P. kuntzei can be found in the Gran Chaco of northern Paraguay and eastern Bolivia to central Argentina.

Sampling methods for all Argentinean populations, except for $P$. argentina and $P$. reptans were those described in previous works (Vilardi et al., 1988). At least ten mother plants were sampled in each population. The samples from United States were collected by Dr. J. C. Evans (Grassland Research Station USDA/ARS). They included five or six mother plants per population. In all cases (North and South American species) the sampled trees were separated at least $50 \mathrm{~m}$ from each other. This distance between sampled mother plants reduces the probability that they interbreed. About 50 pods were collected from each mother plant. The seeds from each tree were stored in different bags. Similar numbers of seeds from different trees (bags) from each population were sampled for the isozyme analysis. Voucher specimens of each mother tree were taxonomically identified and deposited in the Herbarium of the Laboratorio de Genética, Facultad de Ciencias Exactas y Naturales, Universidad de Buenos Aires, Argentina. P. argentina (kindly donated by Dr. P. Villagra, IADIZA, Mendoza, Argentina) and $P$. reptans population samples involved pods from about 30 mother plants collected in a single bag and only one voucher specimen representative of the whole population was prepared. The rationale for this sampling method is that populations of these species form a continuum where individual shrubs can not be identified. The number of seeds analyzed for each population are listed in Table 2.

\section{Isoenzymatic techniques}

Nine systems were studied using horizontal electrophoresis on polyacrylamide gels: esterase (EST), glutamate oxalacetate transaminase (GOT), amino peptidase (AMP), alcohol dehydrogenase (ADH), superoxide dismutase (SOD), 6-phosphogluconate dehydrogenase (6PGD),

Table 2 - Allelic frequencies and number of seeds analyzed (N) of loci showing variation either within or among populations in the studied species of Prosopis. One additional loci. Amp-1, did not show variation and is not included in the list.

\begin{tabular}{|c|c|c|c|c|c|c|c|c|c|c|c|c|c|c|c|c|c|c|c|c|c|c|c|c|c|}
\hline & \multicolumn{5}{|c|}{ Adh-1 } & \multicolumn{4}{|c|}{ Adh -2} & \multicolumn{5}{|c|}{$S k d-1$} & \multicolumn{9}{|c|}{$I d h-1$} & \multicolumn{2}{|c|}{$I d h-2$} \\
\hline & $(\mathrm{N})$ & $1^{30}$ & $1^{28}$ & $1^{24}$ & $1^{29}$ & $(\mathrm{~N})$ & $2^{20}$ & $2^{17}$ & $2^{14}$ & $(\mathrm{~N})$ & $1^{24}$ & $1^{22}$ & $1^{19}$ & $1^{17}$ & $(\mathrm{~N})$ & $1^{75}$ & $1^{70}$ & $1^{63}$ & $1^{60}$ & $1^{86}$ & $1^{84}$ & $1^{82}$ & $1^{80}$ & (N) & $2^{43}$ \\
\hline P. $r u \mathrm{HE}$ & 68 & 1 & 0 & 0 & 0 & 68 & 0 & 1 & 0 & 66 & 0.15 & 0.85 & 0 & 0 & 63 & 0 & 0.42 & 0.58 & 0 & 0 & 0 & 0 & 0 & 63 & 0 \\
\hline P. $r u \mathrm{SA}$ & 53 & 1 & 0 & 0 & 0 & 53 & 0.01 & 0.99 & 0.00 & 55 & 0.07 & 0.93 & 0 & 0 & 22 & 0 & 0.57 & 0.43 & 0 & 0 & 0 & 0 & 0 & 22 & 0 \\
\hline P. $r u \mathrm{RI}$ & 62 & 1 & 0 & 0 & 0 & 61 & 0.06 & 0.92 & 0.02 & 59 & 0.08 & 0.92 & 0 & 0 & 55 & 0 & 0.39 & 0.61 & 0 & 0 & 0 & 0 & 0 & 55 & 0 \\
\hline P. $r u$ PI & 41 & 0.93 & 0.07 & 0 & 0 & 41 & 0 & 1 & 0 & 102 & 0.14 & 0.28 & 0.58 & 0 & 97 & 0 & 0.61 & 0.37 & 0.02 & 0 & 0 & 0 & 0 & 97 & 0 \\
\hline P. $f l \mathrm{LA}$ & 100 & 0.64 & 0.25 & 0.11 & 0 & 100 & 0 & 1 & 0 & 48 & 0.33 & 0.60 & 0.07 & 0 & 40 & 0.06 & 0.10 & 0.81 & 0.03 & 0 & 0 & 0 & 0 & 40 & 1 \\
\hline P. $f l \mathrm{QU}$ & 49 & 0.49 & 0.39 & 0.12 & 0 & 49 & 0 & 1 & 0 & 46 & 0 & 0.58 & 0.21 & 0.21 & 95 & 0.01 & 0.12 & 0.85 & 0.02 & 0 & 0 & 0 & 0 & 96 & 1 \\
\hline P. $g l \mathrm{WE}$ & 39 & 0.94 & 0.06 & 0 & 0 & 39 & 0 & 1 & 0 & 43 & 0.09 & 0.65 & 0.26 & 0 & 56 & 0.05 & 0.92 & 0.03 & 0 & 0 & 0 & 0 & 0 & 56 & 0 \\
\hline P. $g l \mathrm{LC}$ & 40 & 0.95 & 0.05 & 0 & 0 & 40 & 0 & 1 & 0 & 42 & 0.19 & 0.43 & 0.38 & 0 & 47 & 0.04 & 0.63 & 0.33 & 0 & 0 & 0 & 0 & 0 & 36 & 0 \\
\hline P. $g l \mathrm{BC}$ & 40 & 0.95 & 0.05 & 0 & 0 & 40 & 0 & 1 & 0 & 44 & 0.25 & 0.66 & 0.09 & 0 & 43 & 0.38 & 0.60 & 0.02 & 0 & 0 & 0 & 0 & 0 & 44 & 0 \\
\hline P. $g l \mathrm{FC}$ & 30 & 1 & 0 & 0 & 0 & 30 & 0 & 1 & 0 & 36 & 0.17 & 0.75 & 0.08 & 0 & 45 & 0.21 & 0.61 & 0.18 & 0 & 0 & 0 & 0 & 0 & 46 & 0 \\
\hline P. ve SR & 41 & 0.78 & 0.22 & 0 & 0 & 42 & 0 & 1 & 0 & 32 & 0.53 & 0.47 & 0 & 0 & 42 & 0.83 & 0.17 & 0 & 0 & 0 & 0 & 0 & 0 & 42 & 0 \\
\hline P. $k u \mathrm{HE}$ & 39 & 0 & 1 & 0 & 0 & 39 & 1 & 0 & 0 & 50 & 0 & 1 & 0 & 0 & 50 & 0 & 0 & 0 & 0 & 0 & 0 & 0 & 1 & 50 & 0 \\
\hline P. ar $\mathrm{TI}$ & 64 & 0 & 0 & 0 & 1 & 64 & 1 & 0 & 0 & 47 & 0 & 0.29 & 0.52 & 0.19 & 47 & 0 & 0 & 1 & 0 & 0 & 0 & 0 & 0 & 47 & 1 \\
\hline P. $a r \mathrm{TU}$ & 64 & 0 & 0 & 0 & 1 & 64 & 1 & 0 & 0 & 47 & 0 & 0.16 & 0.65 & 0.19 & 47 & 0 & 0 & 1 & 0 & 0 & 0 & 0 & 0 & 47 & 1 \\
\hline P. re $\mathrm{HE}$ & 50 & 0 & 0 & 0 & 1 & 50 & 0 & 0 & 1 & 40 & 0 & 1 & 0 & 0 & 29 & 0 & 0 & 0 & 0 & 0.22 & 0.45 & 0.33 & 0 & 29 & 0 \\
\hline
\end{tabular}

\begin{tabular}{|c|c|c|c|c|c|c|c|c|c|c|c|c|c|c|c|c|c|c|c|c|c|c|c|c|c|}
\hline & \multicolumn{2}{|c|}{$I d h-2$} & \multicolumn{5}{|c|}{ Est-1 } & \multicolumn{3}{|c|}{ Est -2} & \multicolumn{3}{|c|}{ Est-3 } & \multicolumn{3}{|c|}{ Est -4} & \multicolumn{2}{|c|}{ Est-6 } & \multicolumn{2}{|c|}{ Est-7 } & \multicolumn{2}{|c|}{ Est-8 } & \multicolumn{2}{|c|}{ Est-9 } & \multirow{2}{*}{$\begin{array}{c}\text { Est-10 } \\
(\mathrm{N})\end{array}$} \\
\hline & $2^{26}$ & $2^{45}$ & $(\mathrm{~N})$ & $1^{93}$ & $1^{92}$ & $1^{91}$ & $1^{90}$ & $(\mathrm{~N})$ & $2^{87}$ & $2^{0}$ & (N) & $3^{85}$ & $3^{0}$ & (N) & $4^{81}$ & $4^{0}$ & $(\mathrm{~N})$ & $6^{90}$ & $(\mathrm{~N})$ & $7^{86}$ & (N) & $8^{82}$ & $(\mathrm{~N})$ & $9^{78}$ & \\
\hline P. $r u \mathrm{HE}$ & 1 & 0 & 77 & 0.01 & 0.73 & 0.25 & 0 & 71 & 0.65 & 0.35 & 75 & 0.49 & 0.51 & 76 & 0.24 & 0.76 & 77 & - & 77 & - & 77 & - & 77 & - & 77 \\
\hline P. $r u \mathrm{SA}$ & 1 & 0 & 68 & 0 & 0.89 & 0.11 & 0 & 69 & 0.61 & 0.39 & 69 & 0.79 & 0.21 & 73 & 0.30 & 0.70 & 68 & - & 68 & - & 68 & - & 68 & - & 68 \\
\hline P. ru RI & 1 & 0 & 44 & 0 & 0.89 & 0.11 & 0 & 48 & 0.31 & 0.69 & 49 & 0.87 & 0.13 & 49 & 0.59 & 0.41 & 44 & - & 44 & - & 44 & - & 44 & - & 44 \\
\hline P. ru PI & 1 & 0 & 69 & 0 & 0.93 & 0.07 & 0 & 89 & 0.88 & 0.12 & 89 & 0.70 & 0.30 & 89 & 0.09 & 0.91 & 72 & - & 72 & - & 72 & - & 72 & - & 72 \\
\hline$P . f l \mathrm{LA}$ & 0 & 0 & 96 & 0.17 & 0.52 & 0.32 & 0 & 82 & 0.91 & 0.09 & 87 & 1 & 0 & 87 & 0.88 & 0.12 & 96 & - & 96 & - & 96 & - & 96 & - & 96 \\
\hline P. $f l \mathrm{QU}$ & 0 & 0 & 84 & 0 & 0.83 & 0.16 & 0.01 & 49 & 0.78 & 0.22 & 49 & 0.78 & 0.22 & 50 & 0.50 & 0.50 & 84 & - & 84 & - & 84 & - & 84 & - & 84 \\
\hline P. $g l \mathrm{WE}$ & 1 & 0 & 64 & 0.09 & 0.47 & 0.44 & 0 & 32 & 0.75 & 0.25 & 32 & 0.39 & 0.61 & 31 & 0.18 & 0.82 & 64 & - & 64 & - & 64 & - & 64 & - & 64 \\
\hline P. $g l \mathrm{LC}$ & 1 & 0 & 30 & 0.07 & 0.80 & 0.13 & 0 & 34 & 0.75 & 0.25 & 34 & 0.16 & 0.84 & 34 & 0.03 & 0.97 & 56 & - & 56 & - & 56 & - & 56 & - & 56 \\
\hline P. $g l \mathrm{BC}$ & 1 & 0 & 76 & 0 & 0.26 & 0.74 & 0 & 38 & 1 & 0 & 48 & 0.43 & 0.57 & 38 & 0.03 & 0.97 & 76 & - & 76 & - & 76 & - & 76 & - & 76 \\
\hline P. $g l \mathrm{FC}$ & 1 & 0 & 43 & 0.50 & 0.35 & 0.01 & 0.14 & 52 & 0.67 & 0.33 & 52 & 0.43 & 0.57 & 52 & 0.13 & 0.87 & 43 & - & 43 & - & 43 & - & 43 & - & 43 \\
\hline P. ve SR & 1 & 0 & 31 & 0.10 & 0.74 & 0.16 & 0 & 38 & 0.35 & 0.65 & 38 & 0.24 & 0.76 & 38 & 0.49 & 0.51 & 31 & - & 31 & - & 31 & - & 31 & - & 31 \\
\hline P. $k u \mathrm{HE}$ & 1 & 0 & 50 & 0 & 1 & 0 & 0 & 47 & 0 & 1 & 47 & 0 & 1 & 47 & 0 & 1 & 50 & - & 50 & - & 50 & - & 50 & - & 50 \\
\hline P. ar TI & 0 & 0 & 32 & - & - & - & - & 32 & - & - & 32 & - & - & 32 & - & - & 32 & - & 32 & - & 32 & - & 32 & - & 32 \\
\hline P. ar $\mathrm{TU}$ & 0 & 0 & 32 & - & - & - & - & 32 & - & - & 32 & - & - & 32 & - & - & 32 & - & 32 & - & 32 & - & 32 & - & 32 \\
\hline P. re HE & 0 & 1 & 50 & - & - & - & - & 50 & - & - & 50 & - & - & 50 & - & - & 50 & 1 & 50 & 1 & 50 & 1 & 50 & 1 & 50 \\
\hline
\end{tabular}


Table 2 (cont.)

\begin{tabular}{|c|c|c|c|c|c|c|c|c|c|c|c|c|c|c|c|c|c|c|c|c|c|c|c|c|c|}
\hline & \multicolumn{2}{|c|}{$I d h-2$} & \multicolumn{5}{|c|}{ Est-1 } & \multicolumn{3}{|c|}{ Est-2 } & \multicolumn{3}{|c|}{ Est-3 } & \multicolumn{3}{|c|}{ Est-4 } & \multicolumn{2}{|c|}{ Est-6 } & \multicolumn{2}{|c|}{ Est-7 } & \multicolumn{2}{|c|}{ Est-8 } & \multicolumn{2}{|c|}{ Est-9 } & \multirow{2}{*}{\begin{tabular}{|c} 
Est-10 \\
$(\mathrm{N})$ \\
\end{tabular}} \\
\hline & $2^{26}$ & $2^{45}$ & $(\mathrm{~N})$ & $1^{93}$ & $1^{92}$ & $1^{91}$ & $1^{90}$ & $(\mathrm{~N})$ & $2^{87}$ & $2^{0}$ & (N) & $3^{85}$ & $3^{0}$ & $(\mathrm{~N})$ & $4^{81}$ & $4^{0}$ & $(\mathrm{~N})$ & $6^{90}$ & $(\mathrm{~N})$ & $7^{86}$ & $(\mathrm{~N})$ & $8^{82}$ & $(\mathrm{~N})$ & $9^{78}$ & \\
\hline P. $r u \mathrm{HE}$ & 1 & 0 & 77 & 0.01 & 0.73 & 0.25 & 0 & 71 & 0.65 & 0.35 & 75 & 0.49 & 0.51 & 76 & 0.24 & 0.76 & 77 & - & 77 & - & 77 & - & 77 & - & 77 \\
\hline P. $r u \mathrm{SA}$ & 1 & 0 & 68 & 0 & 0.89 & 0.11 & 0 & 69 & 0.61 & 0.39 & 69 & 0.79 & 0.21 & 73 & 0.30 & 0.70 & 68 & - & 68 & - & 68 & - & 68 & - & 68 \\
\hline P. $r u$ RI & 1 & 0 & 44 & 0 & 0.89 & 0.11 & 0 & 48 & 0.31 & 0.69 & 49 & 0.87 & 0.13 & 49 & 0.59 & 0.41 & 44 & - & 44 & - & 44 & - & 44 & - & 44 \\
\hline P. $r u$ PI & 1 & 0 & 69 & 0 & 0.93 & 0.07 & 0 & 89 & 0.88 & 0.12 & 89 & 0.70 & 0.30 & 89 & 0.09 & 0.91 & 72 & - & 72 & - & 72 & - & 72 & - & 72 \\
\hline P. $f l \mathrm{LA}$ & 0 & 0 & 96 & 0.17 & 0.52 & 0.32 & 0 & 82 & 0.91 & 0.09 & 87 & 1 & 0 & 87 & 0.88 & 0.12 & 96 & - & 96 & - & 96 & - & 96 & - & 96 \\
\hline P. $f l \mathrm{QU}$ & 0 & 0 & 84 & 0 & 0.83 & 0.16 & 0.01 & 49 & 0.78 & 0.22 & 49 & 0.78 & 0.22 & 50 & 0.50 & 0.50 & 84 & - & 84 & - & 84 & - & 84 & - & 84 \\
\hline P. $g l \mathrm{WE}$ & 1 & 0 & 64 & 0.09 & 0.47 & 0.44 & 0 & 32 & 0.75 & 0.25 & 32 & 0.39 & 0.61 & 31 & 0.18 & 0.82 & 64 & - & 64 & - & 64 & - & 64 & - & 64 \\
\hline P. $g l \mathrm{LC}$ & 1 & 0 & 30 & 0.07 & 0.80 & 0.13 & 0 & 34 & 0.75 & 0.25 & 34 & 0.16 & 0.84 & 34 & 0.03 & 0.97 & 56 & - & 56 & - & 56 & - & 56 & - & 56 \\
\hline P. $g l \mathrm{BC}$ & 1 & 0 & 76 & 0 & 0.26 & 0.74 & 0 & 38 & 1 & 0 & 48 & 0.43 & 0.57 & 38 & 0.03 & 0.97 & 76 & - & 76 & - & 76 & - & 76 & - & 76 \\
\hline P. $g l \mathrm{FC}$ & 1 & 0 & 43 & 0.50 & 0.35 & 0.01 & 0.14 & 52 & 0.67 & 0.33 & 52 & 0.43 & 0.57 & 52 & 0.13 & 0.87 & 43 & - & 43 & - & 43 & - & 43 & - & 43 \\
\hline P. ve SR & 1 & 0 & 31 & 0.10 & 0.74 & 0.16 & 0 & 38 & 0.35 & 0.65 & 38 & 0.24 & 0.76 & 38 & 0.49 & 0.51 & 31 & - & 31 & - & 31 & - & 31 & - & 31 \\
\hline P. $k u \mathrm{HE}$ & 1 & 0 & 50 & 0 & 1 & 0 & 0 & 47 & 0 & 1 & 47 & 0 & 1 & 47 & 0 & 1 & 50 & - & 50 & - & 50 & - & 50 & - & 50 \\
\hline P. $a r \mathrm{TI}$ & 0 & 0 & 32 & - & - & - & - & 32 & - & - & 32 & - & - & 32 & - & - & 32 & - & 32 & - & 32 & - & 32 & - & 32 \\
\hline P. $a r \mathrm{TU}$ & 0 & 0 & 32 & - & - & - & - & 32 & - & - & 32 & - & - & 32 & - & - & 32 & - & 32 & - & 32 & - & 32 & - & 32 \\
\hline P. re $\mathrm{HE}$ & 0 & 1 & 50 & - & - & - & - & 50 & - & - & 50 & - & - & 50 & - & - & 50 & 1 & 50 & 1 & 50 & 1 & 50 & 1 & 50 \\
\hline
\end{tabular}

\begin{tabular}{|c|c|c|c|c|c|c|c|c|c|c|c|c|c|c|c|c|c|c|c|c|c|c|c|c|c|}
\hline & \multirow{2}{*}{\begin{tabular}{|c|} 
Est-10 \\
$10^{57}$
\end{tabular}} & \multicolumn{2}{|c|}{ Est-11 } & \multicolumn{2}{|c|}{ Est-12 } & \multicolumn{2}{|c|}{ Est-13 } & \multicolumn{2}{|c|}{ Est-14 } & \multicolumn{2}{|c|}{ Est-15 } & \multicolumn{2}{|c|}{ Amp-1 } & \multicolumn{4}{|c|}{ Amp-2 } & \multicolumn{4}{|c|}{ Amp-3 } & \multicolumn{2}{|c|}{ Sod-1 } & \multicolumn{2}{|c|}{ Sod-2 } \\
\hline & & $(\mathrm{N})$ & $11^{46}$ & (N) & $12^{40}$ & $(\mathrm{~N})$ & $13^{30}$ & $(\mathrm{~N})$ & $14^{20}$ & (N) & $15^{18}$ & $(\mathrm{~N})$ & $1^{100}$ & $(\mathrm{~N})$ & 288 & 276 & 270 & $(\mathrm{~N})$ & 377 & 374 & 371 & $(\mathrm{~N})$ & 191 & (N) & $2^{83}$ \\
\hline P. $r u \mathrm{HE}$ & - & 77 & - & 77 & 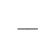 & 77 & 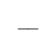 & 77 & - & 77 & - & 41 & 1 & 41 & 0.48 & 0.40 & 0.12 & 41 & - & - & - & 50 & 1 & 50 & 1 \\
\hline P. $r u \mathrm{SA}$ & - & 68 & - & 68 & - & 68 & - & 68 & - & 68 & - & 95 & 1 & 95 & 0.25 & 0.52 & 0.23 & 95 & - & - & - & 50 & 1 & 50 & 1 \\
\hline P. $r u \mathrm{RI}$ & - & 44 & - & 44 & - & 44 & - & 44 & - & 44 & - & 62 & 1 & 62 & 0.39 & 0.41 & 0.20 & 62 & - & - & - & 50 & 1 & 50 & 1 \\
\hline P. $r u$ PI & - & 72 & - & 72 & - & 72 & - & 72 & - & 72 & - & 29 & 1 & 29 & 0.33 & 0.55 & 0.12 & 29 & - & - & - & 50 & 1 & 50 & 1 \\
\hline P. $f l \mathrm{LA}$ & - & 96 & - & 96 & - & 96 & - & 96 & - & 96 & - & 105 & 1 & 105 & 0.25 & 0.57 & 0.18 & 105 & - & - & - & 50 & 1 & 50 & 1 \\
\hline P. $f l \mathrm{QU}$ & - & 84 & - & 84 & - & 84 & - & 84 & - & 84 & - & 44 & 1 & 44 & 0.45 & 0.55 & 0 & 44 & - & - & - & 50 & 1 & 50 & 1 \\
\hline P. $g l \mathrm{WE}$ & - & 64 & - & 64 & - & 64 & - & 64 & - & 64 & - & 30 & 1 & 30 & 0.42 & 0.47 & 0.11 & 30 & - & - & - & 50 & 1 & 50 & 1 \\
\hline P. $g l \mathrm{LC}$ & - & 56 & - & 56 & - & 56 & - & 56 & - & 56 & - & 37 & 1 & 37 & 0.41 & 0.39 & 0.20 & 37 & - & - & - & 50 & 1 & 50 & 1 \\
\hline P. $g l \mathrm{BC}$ & - & 76 & - & 76 & - & 76 & - & 76 & - & 76 & - & 44 & 1 & 44 & 0.25 & 0.45 & 0.30 & 44 & - & - & - & 50 & 1 & 50 & 1 \\
\hline P. $g l \mathrm{FC}$ & - & 43 & - & 43 & - & 43 & - & 43 & - & 43 & - & 42 & 1 & 42 & 0.38 & 0.55 & 0.07 & 42 & - & - & - & 50 & 1 & 50 & 1 \\
\hline P. ve SR & - & 31 & - & 31 & - & 31 & - & 31 & - & 31 & - & 34 & 1 & 34 & 0.79 & 0.21 & 0 & 34 & - & - & - & 40 & 1 & 40 & 1 \\
\hline P. $k u \mathrm{HE}$ & - & 50 & - & 50 & - & 50 & - & 50 & - & 50 & - & 30 & 1 & 30 & 0 & 0.72 & 0.28 & 30 & - & - & - & 31 & 1 & 31 & - \\
\hline P. $a r \mathrm{TI}$ & - & 32 & 1 & 32 & 1 & 32 & 1 & 32 & 1 & 32 & 1 & 16 & 1 & 16 & 1 & 0 & 0 & 11 & 0 & 0.27 & 0.73 & 32 & 1 & 32 & 1 \\
\hline P. $a r \mathrm{TU}$ & - & 32 & 1 & 32 & 1 & 32 & 1 & 32 & 1 & 32 & 1 & 16 & 1 & 16 & 1 & 0 & 0 & 14 & 0.07 & 0.72 & 0.21 & 32 & 1 & 32 & 1 \\
\hline P. re $\mathrm{HE}$ & 1 & 50 & - & 50 & $\ldots$ & 50 & - & 50 & - & 50 & - & 80 & 1 & 80 & 1 & 0 & 0 & 80 & 0.24 & 0 & 0.76 & 50 & 1 & 50 & 1 \\
\hline
\end{tabular}

\begin{tabular}{|c|c|c|c|c|c|c|c|c|c|c|c|c|c|c|c|c|c|c|c|c|c|c|c|c|}
\hline & \multicolumn{2}{|c|}{ Sod -3} & \multicolumn{2}{|c|}{ Sod-4 } & \multicolumn{2}{|c|}{ Sod-5 } & \multicolumn{2}{|c|}{ Sod-6 } & \multicolumn{2}{|c|}{ Sod-7 } & \multicolumn{2}{|c|}{ Sod- 8} & \multicolumn{5}{|c|}{$P g d-1$} & \multicolumn{4}{|c|}{$P g d-2$} & \multicolumn{3}{|c|}{$\operatorname{Pr} x-1$} \\
\hline & $(\mathrm{N})$ & $3^{75}$ & $(\mathrm{~N})$ & $4^{72}$ & $(\mathrm{~N})$ & $5^{50}$ & $(\mathrm{~N})$ & $6^{70}$ & $(\mathrm{~N})$ & $7^{60}$ & $(\mathrm{~N})$ & $8^{95}$ & $(\mathrm{~N})$ & $1^{30}$ & $1^{26}$ & $1^{23}$ & $1^{0}$ & $(\mathrm{~N})$ & $2^{33}$ & $2^{21}$ & $2^{0}$ & $(\mathrm{~N})$ & $1^{36}$ & $1^{0}$ \\
\hline P. $r u \mathrm{HE}$ & 50 & 1 & 50 & 1 & 50 & 1 & 50 & - & 50 & - & 50 & - & 101 & 0.60 & 0.40 & 0 & 0 & 100 & 0.30 & 0.16 & 0.54 & 49 & 0.10 & 0.90 \\
\hline P. ru SA & 50 & 1 & 50 & 1 & 50 & 1 & 50 & . & 50 & - & 50 & - & 62 & 0.40 & 0.60 & 0 & 0 & 62 & 0.21 & 0.23 & 0.56 & 44 & 0.07 & 0.93 \\
\hline P. $r u \mathrm{RI}$ & 50 & 1 & 50 & 1 & 50 & 1 & 50 & - & 50 & - & 50 & - & 74 & 0.63 & 0.37 & 0 & 0 & 84 & 0.26 & 0.16 & 0.58 & 40 & 0.06 & 0.94 \\
\hline P. $r u$ PI & 50 & 1 & 50 & 1 & 50 & 1 & 50 & . & 50 & . & 50 & - & 78 & 0.14 & 0.86 & 0 & 0 & 79 & 0.19 & 0.20 & 0.61 & 40 & 0.42 & 0.58 \\
\hline P. $f l \mathrm{LA}$ & 50 & 1 & 50 & 1 & 50 & 1 & 50 & - & 50 & - & 50 & - & 41 & 0.78 & 0.22 & 0 & 0 & 41 & 0.06 & 0 & 0.94 & 90 & 0.87 & 0.13 \\
\hline P. $f l \mathrm{QU}$ & 50 & 1 & 50 & 1 & 50 & 1 & 50 & - & 50 & - & 50 & - & 102 & 0.01 & 0.99 & 0 & 0 & 68 & 0.12 & 0.20 & 0.68 & 41 & 0.32 & 0.68 \\
\hline P. $g l \mathrm{WE}$ & 50 & 1 & 50 & 1 & 50 & 1 & 50 & - & 50 & - & 50 & - & 56 & 0 & 1 & 0 & 0 & 56 & 0 & 0 & 1 & 31 & 0.19 & 0.81 \\
\hline P. $g l \mathrm{LC}$ & 50 & 1 & 50 & 1 & 50 & 1 & 50 & - & 50 & - & 50 & - & 36 & 0 & 1 & 0 & 0 & 36 & 0.03 & 0 & 0.97 & 26 & 0.52 & 0.48 \\
\hline P. $g l \mathrm{BC}$ & 50 & 1 & 50 & 1 & 50 & 1 & 50 & - & 50 & - & 50 & - & 46 & 0 & 1 & 0 & 0 & 46 & 0.02 & 0 & 0.98 & 40 & 0.06 & 0.94 \\
\hline P. $g l \mathrm{FC}$ & 50 & 1 & 50 & 1 & 50 & 1 & 50 & - & 50 & - & 50 & - & 32 & 0 & 1 & 0 & 0 & 32 & 0 & 0 & 1 & 38 & 0.07 & 0.93 \\
\hline P. ve SR & 40 & 1 & 40 & 1 & 40 & 1 & 40 & - & 40 & - & 40 & - & 34 & 0 & 1 & 0 & 0 & 34 & 0 & 0 & 1 & 48 & - & - \\
\hline P. $k u \mathrm{HE}$ & 31 & 1 & 31 & 1 & 31 & - & 31 & 1 & 31 & - & 31 & - & 26 & 1 & 0 & 0 & 0 & 26 & 0 & 0 & 1 & 47 & 0.62 & 0.38 \\
\hline P. $a r \mathrm{TI}$ & 32 & 1 & 32 & 1 & 32 & 1 & 32 & - & 32 & 1 & 32 & 1 & 47 & 0.94 & 0.02 & 0 & 0.04 & 47 & 1 & 0 & 0 & 37 & 0.84 & 0.16 \\
\hline P. ar $\mathrm{TU}$ & 32 & 1 & 32 & 1 & 32 & 1 & 32 & - & 32 & 1 & 32 & 1 & 48 & 1 & 0 & 0 & 0 & 48 & 0.99 & 0 & 0.01 & 41 & 0.72 & 0.28 \\
\hline P. re HE & 50 & 1 & 50 & 1 & 50 & 1 & 50 & - & 50 & - & 50 & $=$ & 50 & 1 & 0 & 0 & 0 & 50 & 0 & 0 & 1 & 50 & - & - \\
\hline
\end{tabular}

\begin{tabular}{|c|c|c|c|c|c|c|c|c|c|c|c|c|c|c|c|c|c|c|c|c|c|c|c|c|c|}
\hline & \multicolumn{3}{|c|}{ Prx -2} & \multicolumn{3}{|c|}{ Prx-3 } & \multicolumn{2}{|c|}{ Prx-4 } & \multicolumn{4}{|c|}{ Prx -5} & \multicolumn{2}{|c|}{ Prx-6 } & \multicolumn{2}{|c|}{ Prx -7} & \multicolumn{9}{|c|}{ Got-1 } \\
\hline & $(\mathrm{N})$ & $2^{29}$ & $2^{0}$ & $(\mathrm{~N})$ & $3^{21}$ & $3^{0}$ & $(\mathrm{~N})$ & $4^{40}$ & $(\mathrm{~N})$ & $5^{39}$ & $5^{38}$ & $5^{37}$ & $(\mathrm{~N})$ & $6^{32}$ & $(\mathrm{~N})$ & $7^{25}$ & $(\mathrm{~N})$ & $1^{72}$ & $1^{69}$ & $1^{61}$ & $1^{71}$ & $1^{70}$ & $1^{69,5}$ & $1^{68,5}$ & $1^{68}$ \\
\hline P. $r u \mathrm{HE}$ & 49 & 0.89 & 0.11 & 33 & 0.74 & 0.26 & 33 & - & 33 & - & - & - & 33 & - & 33 & - & 86 & 0.05 & 0.95 & 0 & 0 & 0 & 0 & 0 & 0 \\
\hline P. $r u \mathrm{SA}$ & 44 & 0.47 & 0.53 & 44 & 0.77 & 0.23 & 44 & - & 44 & - & - & - & 44 & - & 44 & - & 52 & 0.11 & 0.82 & 0.07 & 0 & 0 & 0 & 0 & 0 \\
\hline P. ru RI & 40 & 0.72 & 0.28 & 40 & 0.38 & 0.62 & 40 & - & 40 & - & - & - & 40 & - & 40 & - & 49 & 0.05 & 0.95 & 0 & 0 & 0 & 0 & 0 & 0 \\
\hline P. $r u \mathrm{PI}$ & 40 & 0.27 & 0.73 & 40 & 0.29 & 0.71 & 40 & - & 40 & - & - & - & 40 & - & 40 & - & 38 & 0.13 & 0.87 & 0 & 0 & 0 & 0 & 0 & 0 \\
\hline P. $f l \mathrm{LA}$ & 98 & 0.88 & 0.12 & 96 & 0.66 & 0.34 & 96 & - & 96 & - & - & - & 96 & - & 96 & - & 60 & 0.44 & 0.56 & 0 & 0 & 0 & 0 & 0 & 0 \\
\hline P. $f l \mathrm{QU}$ & 41 & 0.72 & 0.28 & 41 & 0.38 & 0.62 & 41 & - & 41 & - & - & - & 41 & - & 41 & - & 64 & 0.51 & 0.44 & 0.05 & 0 & 0 & 0 & 0 & 0 \\
\hline P. $g l \mathrm{WE}$ & 31 & 1 & 0 & 31 & 0.57 & 0.43 & 31 & 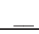 & 31 & & $=$ & $=$ & 31 & & 31 & & 91 & 0.11 & 0.38 & 0 & 0 & 0.51 & 0 & 0 & 0 \\
\hline
\end{tabular}


Table 2 (cont.)

\begin{tabular}{|c|c|c|c|c|c|c|c|c|c|c|c|c|c|c|c|c|c|c|c|c|c|c|c|c|c|}
\hline & \multicolumn{3}{|c|}{$\operatorname{Pr} x-2$} & \multicolumn{3}{|c|}{ Prx $x-3$} & \multicolumn{2}{|c|}{$\operatorname{Pr} x-4$} & \multicolumn{4}{|c|}{ Prx $x-5$} & \multicolumn{2}{|c|}{ Prx-6 } & \multicolumn{2}{|c|}{ Prx -7} & \multicolumn{9}{|c|}{ Got-1 } \\
\hline & $(\mathrm{N})$ & $2^{29}$ & $2^{0}$ & $(\mathrm{~N})$ & $3^{21}$ & $3^{0}$ & $(\mathrm{~N})$ & $4^{40}$ & $(\mathrm{~N})$ & $5^{39}$ & $5^{38}$ & $5^{37}$ & $(\mathrm{~N})$ & $6^{32}$ & $(\mathrm{~N})$ & $7^{25}$ & $(\mathrm{~N})$ & $1^{72}$ & $1^{69}$ & $1^{61}$ & $1^{71}$ & $1^{70}$ & $1^{69,5}$ & $1^{68,5}$ & $1^{68}$ \\
\hline P. $g l \mathrm{LC}$ & 26 & 0.67 & 0.33 & 26 & 0.19 & 0.81 & 26 & - & 26 & - & - & - & 26 & - & 26 & - & 56 & 0.09 & 0.46 & 0 & 0 & 0.45 & 0 & 0 & 0 \\
\hline P. $g l \mathrm{BC}$ & 40 & 0.84 & 0.16 & 40 & 0.28 & 0.72 & 40 & - & 40 & - & - & - & 40 & - & 40 & - & 34 & 0.25 & 0.54 & 0 & 0 & 0.21 & 0 & 0 & 0 \\
\hline P. $g l \mathrm{FC}$ & 38 & 0.72 & 0.28 & 35 & 0.11 & 0.89 & 35 & - & 35 & - & - & - & 35 & - & 35 & - & 39 & 0.20 & 0.35 & 0 & 0 & 0.45 & 0 & 0 & 0 \\
\hline P. ve SR & 48 & - & - & 48 & - & - & 48 & - & 48 & - & - & - & 48 & 1 & 48 & 1 & 43 & 0.12 & 0.08 & 0 & 0.80 & 0 & 0 & 0 & 0 \\
\hline P. $k u \mathrm{HE}$ & 47 & 0.85 & 0.15 & 47 & 0.45 & 0.55 & 47 & - & 47 & - & - & - & 47 & - & 47 & - & 98 & 0 & 0 & 0 & 0 & 0 & 0 & 0 & 0 \\
\hline P. $a r \mathrm{TI}$ & 37 & 0.84 & 0.16 & 37 & 0.27 & 0.73 & 37 & - & 37 & - & - & - & 37 & - & 37 & - & 27 & 0 & 0 & 0 & 0 & 0 & 0.80 & 0.20 & 0 \\
\hline P. ar TU & 41 & 0.60 & 0.40 & 41 & 0.24 & 0.76 & 41 & - & 41 & - & - & - & 41 & - & 41 & - & 30 & 0 & 0 & 0 & 0 & 0 & 0.55 & 0.45 & 0 \\
\hline \multirow{3}{*}{ P. re HE } & 50 & - & 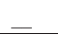 & 50 & & & 50 & 1 & 50 & 0.42 & 0.14 & 0.44 & 50 & 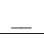 & 50 & & 100 & 0 & 0 & 0 & 0 & 0 & 0 & 0 & 0.05 \\
\hline & \multicolumn{2}{|c|}{ Got-1 } & \multicolumn{10}{|c|}{ Got-2 } & \multicolumn{2}{|c|}{ Got-3 } & \multicolumn{2}{|c|}{ Got-4 } & \multicolumn{2}{|c|}{ Got -5} & \multicolumn{4}{|c|}{ Got-6 } & \multicolumn{3}{|c|}{ Got-7 } \\
\hline & $1^{67}$ & $1^{71,5}$ & $(\mathrm{~N})$ & $2^{48}$ & $2^{40}$ & $2^{34}$ & $2^{27}$ & $2^{54}$ & $2^{39}$ & $2^{37}$ & $2^{38}$ & $2^{36}$ & $(\mathrm{~N})$ & $3^{49}$ & $(\mathrm{~N})$ & $4^{44}$ & $(\mathrm{~N})$ & $5^{50}$ & $(\mathrm{~N})$ & $6^{25}$ & $6^{26}$ & $6^{0}$ & (N) & $7^{24}$ & $7^{22}$ \\
\hline P. $r u \mathrm{HE}$ & 0 & 0 & 61 & 0.59 & 0.27 & 0.08 & 0.06 & 0 & 0 & 0 & 0 & 0 & 86 & - & 86 & - & 86 & - & 86 & 0 & 0 & 1 & 86 & - & - \\
\hline P. $r u \mathrm{SA}$ & 0 & 0 & 47 & 0.80 & 0.20 & 0 & 0 & 0 & 0 & 0 & 0 & 0 & 52 & - & 52 & - & 52 & - & 52 & 0 & 0 & 1 & 52 & - & - \\
\hline P. $r u$ RI & 0 & 0 & 44 & 0.59 & 0.41 & 0 & 0 & 0 & 0 & 0 & 0 & 0 & 49 & - & 49 & - & 49 & - & 49 & 0 & 0 & 1 & 49 & - & - \\
\hline P. $r u$ PI & 0 & 0 & 34 & 0.54 & 0.40 & 0.06 & 0 & 0 & 0 & 0 & 0 & 0 & 38 & - & 38 & - & 38 & - & 38 & 0 & 0 & 1 & 38 & - & - \\
\hline P. $f l \mathrm{LA}$ & 0 & 0 & 51 & 0.28 & 0.72 & 0 & 0 & 0 & 0 & 0 & 0 & 0 & 60 & - & 60 & - & 60 & - & 60 & 0 & 0 & 1 & 60 & - & - \\
\hline$P . f l \mathrm{QU}$ & 0 & 0 & 51 & 0.31 & 0.64 & 0.05 & 0 & 0 & 0 & 0 & 0 & 0 & 51 & - & 51 & - & 64 & - & 64 & 0 & 0 & 1 & 51 & - & - \\
\hline P. $g l \mathrm{WE}$ & 0 & 0 & 55 & 0.63 & 0.37 & 0 & 0 & 0 & 0 & 0 & 0 & 0 & 55 & - & 55 & - & 91 & - & 91 & 0 & 0 & 1 & 55 & - & - \\
\hline P. $g l \mathrm{LC}$ & 0 & 0 & 53 & 0.82 & 0.18 & 0 & 0 & 0 & 0 & 0 & 0 & 0 & 53 & - & 53 & - & 56 & - & 56 & 0 & 0 & 1 & 53 & - & - \\
\hline P. $g l \mathrm{BC}$ & 0 & 0 & 21 & 0.93 & 0.07 & 0 & 0 & 0 & 0 & 0 & 0 & 0 & 34 & - & 34 & - & 34 & - & 34 & 0 & 0 & 1 & 34 & - & - \\
\hline P. $g l \mathrm{FC}$ & 0 & 0 & 36 & 0.90 & 0.10 & 0 & 0 & 0 & 0 & 0 & 0 & 0 & 39 & - & 39 & - & 39 & - & 39 & 0 & 0 & 1 & 39 & - & - \\
\hline P. ve SR & 0 & 0 & 34 & 0 & 0 & 0 & 0 & 1 & 0 & 0 & 0 & 0 & 34 & 1 & 34 & 1 & 43 & - & 43 & 0 & 0 & 1 & 34 & - & - \\
\hline P. $k u \mathrm{HE}$ & 0 & 1 & 82 & 0 & 0 & 0 & 0 & 0 & 0.96 & 0.04 & 0 & 0 & 100 & - & 100 & - & 98 & 1 & 98 & 0.23 & 0.63 & 0.14 & 100 & - & - \\
\hline P. ar TI & 0 & 0 & 39 & 0.42 & 0 & 0.58 & 0 & 0 & 0 & 0 & 0 & 0 & 31 & - & 31 & - & 27 & - & 27 & 0 & 0 & 1 & 31 & 0.84 & 0.16 \\
\hline P. ar $\mathrm{TU}$ & 0 & 0 & 39 & 0.81 & 0.04 & 0.15 & 0 & 0 & 0 & 0 & 0 & 0 & 31 & - & 31 & - & 30 & - & 30 & 0 & 0 & 1 & 39 & 0.83 & 0.17 \\
\hline P. re HE & 0.95 & 0.05 & 100 & 0 & 0 & 0 & 0 & 0 & 0 & 0 & 0.27 & 0.73 & 31 & - & 31 & - & 100 & - & 100 & 0 & 0 & 1 & 31 & - & - \\
\hline
\end{tabular}

peroxidase (PRX), isocitrate dehydrogenase (IDH) and shikimic dehydrogenase (SKD). The methods employed for the former seven systems are described in Saidman (1985). For IDH and SKD the method was adapted from Verga (1995). The homogenates were made from five to seven day old cotyledons for all systems but $\mathrm{ADH}$, for which 16-h-old seedlings were analyzed. Since ADH must be analyzed at a different life stage, it was not possible to study this system on the same individuals used for the assays with all the other enzymes.

\section{Statistical methods}

The genetic interpretation of isozyme data in $P$. argentina and $P$. kuntzei was based on previous studies on other species of Prosopis (Saidman and Vilardi, 1987; Verga, 1995). Standard measures of genetic diversity were calculated for each population from allelic frequency data. The diversity parameters estimated included mean number of alleles per locus $(\hat{A})$, mean number of alleles per polymorphic locus $(\hat{A P})$, percentage of polymorphic loci $(\hat{P})$, and mean expected $\left(\hat{H}_{e}\right)$ and observed $\left(\hat{H}_{o}\right)$ heterozygosity. They were calculated using BIOSYS 1.7 (Swofford and Selander, 1981). Expected heterozygosity estimates were compared among species by Kruskall-Wallis analysis of variance, using the program Statistix ver. 1.0 (Analytical Software, 1996). Pair-wise comparisons of mean heterozygosities were performed by Wilcoxon test using the program Statistica (Statsoft, Inc., 1995). These test have the advantage of having no assumptions about the distribution of the coefficients to be compared.

In order to discuss bias from Hardy-Weinberg expected frequencies, Wright (1951) $\hat{F}_{\text {is }}$ indices were estimated.

Differentiation between populations were estimated by two methods. The first one was based on unbiased Nei's (1978) genetic distances. This method might have some error because the precise homology between genes in species of different sections are uncertain due to the absence of intersectional hybrids. The second approach employs a phenotypic criterion to assess band homologies and to estimate relative associations among species. The bands were considered present when their frequency in the population was equal to or higher than 0.05 and a 0-1 matrix (presence-absence) was constructed. Manhattan distances were estimated from that matrix using the program RAPD (Black IV, 1996). Based on the genetic distances, two phenograms were obtained by UPGMA using the same program. In order to evaluate the reliability of the branches, 100 phenograms were obtained from bootstrapped pseudoreplicates of the respective matrices.

\section{Results}

\section{Allelic frequencies}

From all species, a total of 46 loci were detected. Some of them were invariant: Amp-1, Sod-1, Sod-3, and 
Sod-4. The remaining loci showed variation within or among populations (Table 2).

For every system, the loci were numbered according to the chronological order in which they were described in previous works. Alleles were named with a superscript indicating the relative mobility of the corresponding product. The superscript 0 refers to null alleles. Some loci were species-specific, showing no homology with loci present in other species (Table 2).

\section{Genetic variability and endogamy estimates}

Genetic variability and endogamy estimates are shown in Table 3 . In all populations $\hat{H}_{e}$ was higher than $\hat{H}_{o}$, yielding positive $\hat{F}_{\text {is }}$ estimates. This result indicates that some endogamy occurs in all studied populations. There is no significant correlation between genetic variability and the $\hat{F}_{\text {is }}$ coefficient $(\mathrm{p}=0.45)$. For most variability estimates $P$. flexuosa (Quilmes) exhibited the highest values, while P. kuntzei (Herrera) and P. reptans (Herrera) were the less variable populations.

The estimates of $\hat{A}, \hat{A} P$, and $\hat{H}_{e}$ were compared among populations by Kruskall-Wallis statistics (KWS). Non-significant differences were detected for ÂP $(\mathrm{KWS}=8.36 ; \mathrm{p}=0.87$ ), but the differences among populations were significant for both $\hat{A}(\mathrm{KWS}=28.477, \mathrm{p}=0.012)$ and $\hat{H}_{e}(\mathrm{KWS}=22.536, \mathrm{p}=0.047)$.

When only populations of the same species were included in the analyses of $\hat{A}$ and $\hat{H}_{e}$, the differences were not significant in any case (KWS $=0.008-0.31, \mathrm{p}=0.93-0.96$ and KWS $=0.026-0.43, \mathrm{p}=0.872-0.509$ for $\hat{\mathrm{A}}$ and $\hat{H}_{e}$, respectively). All populations of each species were pooled and the comparisons were made at species level. The Kruskall-Wallis test indicated highly significant differences for both $\hat{A}$ and $\hat{H}_{e}(\mathrm{KWS}=27.89, \mathrm{p}=0.0001$ and $\mathrm{KWS}=28.20, \mathrm{p}=0.0001$ respectively). In general terms the species of section Algarobia resulted in more variation than the rest $(\bar{A}=1.71, \bar{H} e=0.20)$. However, when Algarobia species were compared to each other the differences were significant (KWS $=12.64, \mathrm{p}=0.013$ and KWS $=11.92, \mathrm{p}=0.018$ for $\hat{A}$ and $\hat{H}_{e}$ respectively) as a consequence of the low variability of $\mathrm{P}$. kuntzei (Herrera). When this population was excluded, no significant differences occurred among species of Algarobia $(\mathrm{KWS}=2.98$, $\mathrm{p}=0.39$ and $\mathrm{KWS}=4.73, \mathrm{p}=0.19$ for $\hat{A}$ and $\hat{H}_{e}$, respectively). When P. kuntzei (Herrera), $P$. argentina, and $P$. reptans (Herrera) were compared, the differences were not significant $(\mathrm{KWS}=1.46, \mathrm{p}=0.50$ and $\mathrm{KWS}=0.84$, $\mathrm{p}=0.65$ for $\hat{A}$ and $\hat{H}_{e}$, respectively).

The proportion of polymorphic loci $(\hat{P})$ differed significantly among populations and among species $\left(\chi_{14}^{2}=28.84, P=0.011\right.$ and $\left.\chi_{6}^{2}=28.21, \mathrm{p}<0.0001\right)$. The proportion of polymorphic loci was compared among the same population groups as described for $\hat{A}$ and $\hat{H}_{e}$. The species of Algarobia studied here exhibited significant differences for $\mathrm{P}$ estimates $\left(\chi^{2}{ }_{4}=11.92, \mathrm{p}=0.018\right)$, but these differences became non-significant when $P$. kuntzei (Herrera) was excluded $\left(\chi_{3}^{2}=3.75, \mathrm{p}=0.29\right)$. The $P$ estimates did not differ statistically among $P$. kuntzei

Table 3 - Genetic variability and fixation index $\left(F_{I S}\right)$ coefficients estimated for each population. $\mathrm{N}=$ mean sample size per locus, $\hat{A}=$ mean number of alleles per locus, $\hat{A P}=$ mean number of alleles per polymorphic locus, $\hat{P}=\%$ of polymorphic loci $(5 \%$ criterion $), \hat{H}_{o}=$ observed heterozygosity, $\hat{H}_{e}=$ unbiased heterozygosity expected under Hardy-Weinberg. Numbers in parenthesis indicate the standard error.

\begin{tabular}{|c|c|c|c|c|c|c|c|}
\hline Population & $\mathrm{N}$ & $\hat{A}$ & $\hat{A P}$ & $\hat{P}$ & $\hat{H}_{o}$ & $\hat{H}_{e}$ & $\vec{F}_{i s}$ \\
\hline P. argentina Tinogasta & $34.4(1.4)$ & $1.39(0.63)$ & $2.22(0.44)$ & $31.03(8.74)$ & $0.038(0.019)$ & $0.110(0.03)$ & $0.415(0.189)$ \\
\hline$P$. argentina Tucunuco & $35.5(1.4)$ & $1.43(0.69)$ & $2.33(0.50)$ & $31.03(8.74)$ & $0.036(0.017)$ & $0.116(0.04)$ & $0.193(0.165)$ \\
\hline P. reptans Herrera & $53.9(2.6)$ & $1.28(0.62)$ & $2.40(0.55)$ & $20.00(8.16)$ & $0.025(0.014)$ & $0.085(0.04)$ & $0.209(0.114)$ \\
\hline P. kuntzei Herrera & $50.3(3.2)$ & $1.25(0.44)$ & $2.00(0.00)$ & $25.00(9.03)$ & $0.006(0.005)$ & $0.094(0.04)$ & $0.540(0.115)$ \\
\hline P. ruscifolia Herrera & $64.4(2.8)$ & $1.79(0.83)$ & $2.36(0.63)$ & $58.33(10.28)$ & $0.057(0.022)$ & $0.233(0.05)$ & $0.294(0.127)$ \\
\hline P. ruscifolia Sarmiento & $57.4(2.3)$ & $1.71(0.69)$ & $2.21(0.43)$ & $59.33(10.28)$ & $0.049(0.020)$ & $0.226(0.05)$ & $0.335(0.087)$ \\
\hline P. ruscifolia Rivadavia & $49.7(1.3)$ & $1.75(0.68)$ & $2.20(0.41)$ & $62.50(10.09)$ & $0.052(0.020)$ & $0.225(0.05)$ & $0.390(0.107)$ \\
\hline P. ruscifolia Pinto & $57.0(3.1)$ & $1.83(0.76)$ & $2.33(0.49)$ & $62.50(10.09)$ & $0.045(0.017)$ & $0.234(0.05)$ & $0.476(0.091)$ \\
\hline P. flexuosa La Amarga & $76.4(3.4)$ & $1.83(0.87)$ & $2.43(0.65)$ & $58.33(10.28)$ & $0.082(0.027)$ & $0.217(0.04)$ & $0.096(0.065)$ \\
\hline P. flexuosa Quilmes & $60.7(2.8)$ & $1.87(0.85)$ & $2.50(0.52)$ & $58.33(10.28)$ & $0.056(0.020)$ & $0.262(0.05)$ & $0.395(0.085)$ \\
\hline P. glandulosa Weslaco & $51.0(2.5)$ & $1.71(0.81)$ & $2.42(0.51)$ & $50.00(10.42)$ & $0.045(0.019)$ & $0.209(0.05)$ & $0.379(0.102)$ \\
\hline P. glandulosa La Copita & $44.4(1.6)$ & $1.79(0.78)$ & $2.36(0.50)$ & $58.33(10.28)$ & $0.056(0.022)$ & $0.215(0.05)$ & $0.165(0.094)$ \\
\hline P. glandulosa Bell Co. & $50.0(2.3)$ & $1.71(0.75)$ & $2.31(0.48)$ & $54.17(10.39)$ & $0.049(0.019)$ & $0.178(0.05)$ & $0.269(0.129)$ \\
\hline P. glandulosa Frio Co. & $41.9(0.9)$ & $1.75(0.90)$ & $2.50(0.67)$ & $50.00(10.43)$ & $0.062(0.025)$ & $0.203(0.05)$ & $0.070(0.070)$ \\
\hline P. velutina Santa Rita & $37.9(0.9)$ & $1.56(0.82)$ & $2.40(0.70)$ & $40.00(10.00)$ & $0.038(0.016)$ & $0.142(0.04)$ & $0.312(0.175)$ \\
\hline
\end{tabular}


(Herrera), $P$. reptans (Herrera), and $P$. argentina populations $\left(\chi_{2}^{2}=1.38, \mathrm{p}=0.50\right)$.

\section{Genetic distances among populations}

Two matrices of genetic distances (Table 4) were obtained respectively from Manhattan and Nei's genetic distances. They were highly consistent according to a Mantel test $(\mathrm{r}=0.95 ; \mathrm{p}=0)$ based on 500 permutations. The corresponding trees are also highly consistent with minor differences. The phenograms are rather consistent with morphology because the populations of each species cluster together; species belonging to the section Algarobia are clustered in a single group; and $P$. kuntzei, which is morphologically very different from the remaining species studied of Algarobia, is the most phenetically distant species of this section. Excluding P. kuntzei, $P$. velutina (Arizona, USA) is the most differentiated of Algarobia, and the cluster formed by the Argentinean species $P$. ruscifolia and $P$. flexuosa with the North American P. glandulosa (Figure 2).

The distance between the populations of $P$. argentina and $P$. reptans Herrera was almost as high as that recorded among any of the Algarobia species.

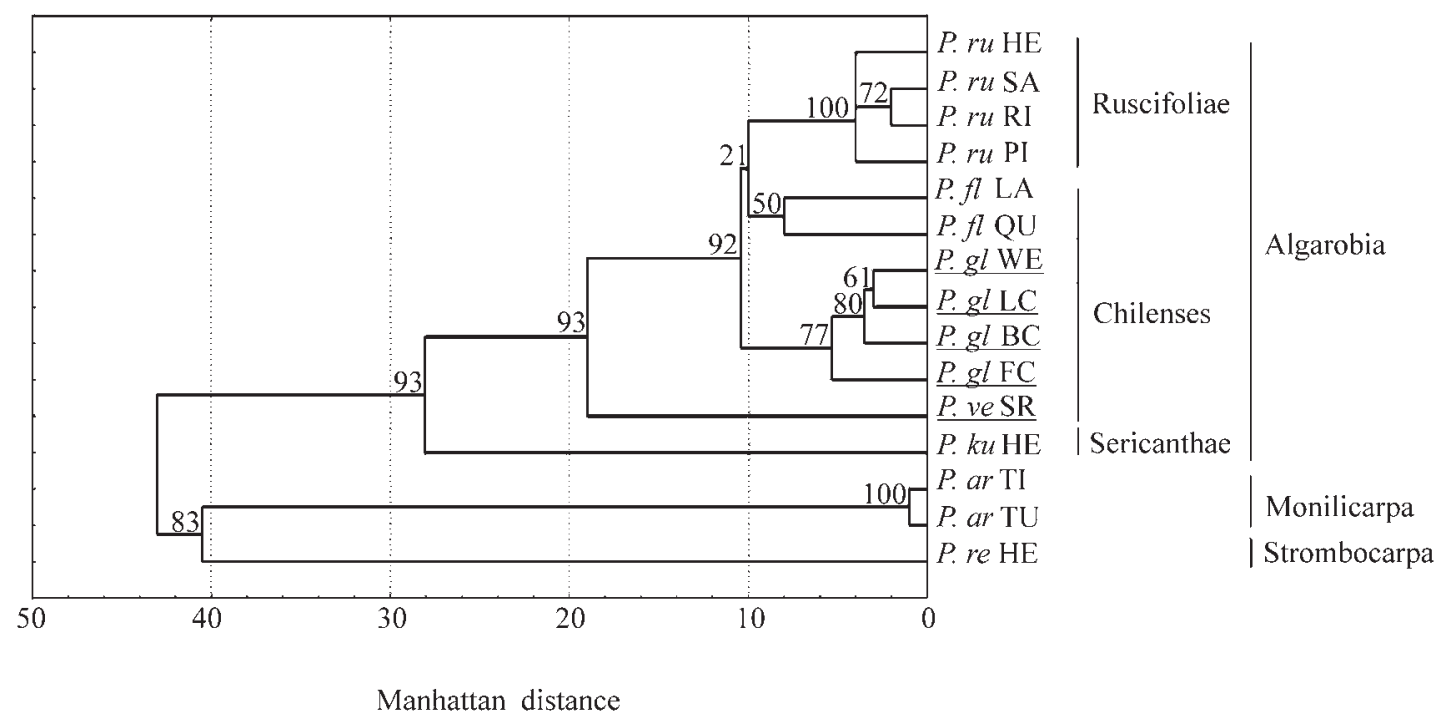

Figure 2 - Phenogram representative of Manhattan distances between populations of the species studied of genus Prosopis. Numbers over branches represent bootstrap support for each node. North-American populations are underlined.

Table 4 - Nei (above diagonal) and Manhattan (below diagonal) genetic distance matrix.

\begin{tabular}{|c|c|c|c|c|c|c|c|c|c|c|c|c|c|c|c|}
\hline & $\begin{array}{c}\text { P. ru } \\
\text { HE }\end{array}$ & $\begin{array}{c}\text { P. ru } \\
\text { SA }\end{array}$ & $\begin{array}{c}\text { P. ru } \\
\text { RI }\end{array}$ & $\begin{array}{c}\text { P. ru } \\
\text { PI }\end{array}$ & $\begin{array}{l}P . f l \\
\text { LA }\end{array}$ & $\begin{array}{l}P . f l \\
\text { QU }\end{array}$ & $\begin{array}{l}P . g l \\
\text { WE }\end{array}$ & $\begin{array}{c}P . g l \\
\text { LC }\end{array}$ & $\begin{array}{c}P . g l \\
\text { BC }\end{array}$ & $\begin{array}{c}P . g l \\
\text { FC }\end{array}$ & $\begin{array}{c}P . v e \\
\text { SR }\end{array}$ & $\begin{array}{c}P . k u \\
\text { TA }\end{array}$ & $\begin{array}{c}P . a r \\
\text { TI }\end{array}$ & $\begin{array}{c}P . a r \\
\mathrm{TU}\end{array}$ & $\begin{array}{c}\text { P. re } \\
\mathrm{He}\end{array}$ \\
\hline P. ru HE & & 0.010 & 0.014 & 0.036 & 0.078 & 0.063 & 0.031 & 0.041 & 0.040 & 0.041 & 0.223 & 0.290 & 0.491 & 0.488 & 0.452 \\
\hline P. ru SA & 4.00 & & 0.013 & 0.025 & 0.086 & 0.061 & 0.036 & 0.043 & 0.042 & 0.038 & 0.212 & 0.306 & 0.541 & 0.526 & 0.462 \\
\hline P. ru RI & 4.00 & 2.00 & & 0.040 & 0.074 & 0.061 & 0.051 & 0.057 & 0.061 & 0.048 & 0.213 & 0.295 & 0.509 & 0.500 & 0.445 \\
\hline P. ru PI & 4.00 & 4.00 & 4.00 & & 0.095 & 0.055 & 0.038 & 0.021 & 0.040 & 0.037 & 0.197 & 0.328 & 0.532 & 0.515 & 0.476 \\
\hline$P . f l \mathrm{LA}$ & 12.00 & 10.00 & 10.00 & 8.00 & & 0.037 & 0.104 & 0.112 & 0.118 & 0.119 & 0.301 & 0.351 & 0.500 & 0.504 & 0.534 \\
\hline$P . f l \mathrm{QU}$ & 12.00 & 10.00 & 10.00 & 8.00 & 8.00 & & 0.068 & 0.068 & 0.078 & 0.072 & 0.233 & 0.342 & 0.482 & 0.479 & 0.485 \\
\hline$P . g l \mathrm{WE}$ & 12.00 & 10.00 & 10.00 & 8.00 & 8.00 & 12.00 & & 0.016 & 0.013 & 0.015 & 0.206 & 0.307 & 0.543 & 0.570 & 0.473 \\
\hline$P . g l \mathrm{LC}$ & 11.00 & 9.00 & 9.00 & 7.00 & 9.00 & 11.00 & 3.00 & & 0.022 & 0.015 & 0.189 & 0.296 & 0.503 & 0.492 & 0.453 \\
\hline P. $g l \mathrm{BC}$ & 13.00 & 11.00 & 11.00 & 9.00 & 11.00 & 13.00 & 3.00 & 4.00 & & 0.015 & 0.199 & 0.330 & 0.565 & 0.553 & 0.472 \\
\hline P. $g l \mathrm{FC}$ & 12.00 & 10.00 & 10.00 & 10.00 & 10.00 & 14.00 & 4.00 & 5.0 & 7.00 & & 0.181 & 0.313 & 0.537 & 0.524 & 0.438 \\
\hline P. ve $\mathrm{SR}$ & 22.00 & 20.00 & 20.00 & 20.00 & 20.00 & 22.00 & 14.00 & 17.00 & 17.00 & 18.00 & & 0.478 & 0.760 & 0.743 & 0.561 \\
\hline P. $k u$ TA & 27.00 & 27.00 & 25.00 & 27.00 & 31.00 & 31.00 & 27.00 & 26.00 & 26.00 & 29.00 & 33.00 & & 0.667 & 0.676 & 0.616 \\
\hline P. ar TI & 38.00 & 40.00 & 38.00 & 38.00 & 38.00 & 36.00 & 44.00 & 41.00 & 41.00 & 42.00 & 52.00 & 37.00 & & 0.012 & 0.666 \\
\hline P. ar TU & 39.00 & 41.00 & 39.00 & 39.00 & 39.00 & 37.00 & 45.00 & 42.00 & 42.00 & 43.00 & 53.00 & 38.00 & 1.00 & & 0.665 \\
\hline P. re $\mathrm{HE}$ & 47.00 & 47.00 & 47.00 & 49.00 & 49.00 & 49.00 & 49.00 & 48.00 & 46.00 & 49.00 & 47.00 & 40.00 & 41.00 & 40.00 & \\
\hline
\end{tabular}




\section{Discussion}

According to the differences in genetic variability, two heterogeneous groups of species can be established. The first one, with high variability, involves all species studied of section Algarobia except for P. kuntzei. The second one, with low variability, is represented by the remaining species.

The causes for the differences in genetic variability may be related to the reproductive system, the adaptive strategies or the evolutionary history of these species. The species of Algarobia with high variability were largely considered outcrosser, with self-incompatibility system (Solbrig and Bawa, 1975; Solbrig and Cantilo, 1975; Neff et al., 1977; Simpson, 1977; Simpson et al., 1977; Hunziker et al., 1986). Recent studies on the mating system of $P$. alba, P. nigra, P. flexuosa, P. glandulosa, P. velutina, $P$. ruscifolia, and P.chilensis (Bessega et al., 2000b) indicated that they are mostly outcrosser, although about $15 \%$ selfing can occur. Besides, these species are widely distributed and able to grow in diverse soils (Burkart, 1976). Finally, Bessega et al. (2000a) advanced the hypothesis that hybridization may have played a role in the first steps of species diversification of this group promoting reticulate evolution and boosting invasiveness ability. Information about the mating system of the remaining species is lacking. $P$. argentina is endemic and restricted to the western Argentinean provinces of Catamarca, La Rioja, and Mendoza, and, in contrast with most species of Algarobia, P. argentina shows a marked preference for soft and sandy soils (Burkart, 1976). Consequently, it can be considered a highly specialized species and its low variability a consequence of this high specialization. The low variability of $P$. reptans, as well as other species of the section Strombocarpa, had been previously recorded by Saidman et al., (1996). Selfing in species of Strombocarpa can not be ruled out because at least one species of this section, $P$. tamarugo, appears to self (Hunziker et al., 1986). $P$. reptans was shown to be able to undergo vegetative reproduction by means of underground runners (Burkart, 1976; Roig, 1993). The ability to reproduce vegetatively and the possibility of selfing allow the advancement of the hypothesis that the low variability in $P$. reptans might be the result of founder effect associated with colonization and a certain degree of endogamy (Saidman et al., 1996). However, the estimated $F_{I S}$ does not suggest a higher tendency to selfing than the rest of the studied species of Prosopis.

Populations of $P$. kuntzei are usually dense and widely distributed. Therefore, low population sizes are not a likely explanation for its low variability. Its ability to colonize is similar to that of other species of Algarobia, but unlike the others, P. kuntzei apparently is not involved in natural hybridization events. The evolutionary history of this species might be very different from that of the remaining species of the same section. If hybridization did not play a role in the early evolution of this species, its variability might have been rapidly eroded. The actual cause for the low variability in P. kuntzei should be addressed through deeper analyses of its mating system.

The analysis of isoenzymatic patterns indicated that $P$. argentina, $P$. reptans, $P$. kuntzei, and $P$. velutina can be differentiated from each other and from the rest of the species studied here. The remaining species, which belong to section Algarobia, exhibit, for most enzyme loci, transspecies polymorphisms (similar to those described for DNA sequences in Klein, 1980; Garrigan and Hedrick, 2003), differing from each other only in allelic frequencies. The similarities obtained from isozyme data agree with the expected relationships among the three sections based on morphological grounds (Burkart, 1976). P. argentina (Monilicarpa) and P. reptans (Strombocarpa) are clearly differentiated from each other and from species of Algarobia. This result is consistent with studies based on phenolic compounds (Carman, 1973) and seed proteins (Burghardt and Palacios, 1997). The high biochemical differentiation observed among species belonging to different sections supports the hypothesis that these groups are natural and that the sections in the Burkart's (1976) system might be elevated to subgenera as suggested by Hunziker et al., (1986), Saidman et al., (1996), and Burghardt and Palacios (1997).

Among the species of Algarobia studied here $P$. kuntzei was the most differentiated isoenzymatically. This result is also consistent with morphological data because this species and $P$. sericantha are subaphyllous and horrid trees or shrubs with all branchlets spiny, included in a separate series, Sericanthae. Besides, although natural hybridization is very frequent between species of Algarobia belonging to different series (Palacios and Bravo, 1981; Hunziker et al., 1986), no hybrids have been described involving P. kuntzei. In the present paper, P. kuntzei shows private isoenzymatic patterns, genetic variability significantly lower than the remaining species of Algarobia, and the degree of genetic differentiation with respect to the remaining species of Algarobia is almost as high as those recorded for $P$. reptans (Strombocarpa) or $P$. argentina (Monilicarpa). This evidence suggests that $P$. kuntzei might be included in a section different from that involving the rest of Algarobia species studied here.

According to the present results, the series Chilenses and Ruscifoliae defined by Burkart (1976) would not be natural groups, because the series are not represented in the clusters retrieved. Since the genetic differentiation among these species is low, all of them might be included in a single series rather than two. P. velutina is the most differentiated species within this group. This result suggests that this species has no common origin with the other North American species, $P$. glandulosa. In agreement with previous RAPD and isoenzymatic studies (Bessega et al., 2000c) and cladistic analyses based on cpDNA and rDNA data (Bessega, 2001), the present results suggest that $P$. 
glandulosa and P. velutina would have originated from two independent founder events.

\section{Acknowledgments}

We wish to express our gratitude to Ing. Hugo Cordo and Dr. J. C. Evans for his invaluable help in obtaining samples from the USA and to Prof. R. A. Palacios for taxonomic identification of Argentinean species. Special thanks to Dr. P. Villagra and Ing. $M$. Cony who generously provided the samples of $P$. argentina. This work was carried out thanks to the financial support of the Universidad de Buenos Aires (PID N ${ }^{\circ}$ X086), Agencia Nacional de Promoción Científica y Tecnológica (PICT $\mathrm{N}^{\circ}$ 06628) and Consejo Nacional de Investigaciones Científicas y Técnicas (PIP 0722/98).

\section{References}

Analytical Software (1996) Statistix ver. 1.0: User's Manual. Analytical Software. Tallahassee, FL.

Bessega C (2001) Estructura poblacional y relaciones filogenéticas (distancia y parsimonia) en especies del género Prosopis (Leguminosae). PhD Thesis, FCEyN. Universidad de Buenos Aires, Buenos Aires, Argentina.

Bessega C, Ferreyra LI, Saidman BO and Vilardi JC (2000)a Unexpected low genetic differentiation among allopatric species of section Algarobia of Prosopis (Leguminosae). Genetica 109:255-266.

Bessega C, Ferreyra LI, Julio N, Montoya S, Saidman BO and Vilardi JC (2000)b Mating system parameters in species of genus Prosopis (Leguminosae). Hereditas 132:19-27.

Bessega C, Saidman BO and Vilardi JC (2000)c Isozyme and RAPD studies in Prosopis glandulosa and P. velutina (Leguminosae, Mimosoideae). Genetics and Molecular Biology $23: 1-5$.

Black IV WC (1996) FORTRAN programs for the analysis of RAPD-PCR markers in populations. Colorado State University, Ft. Collins, CO, USA.

Burghardt AD and Palacios RA (1997) Electrophoretic characterization of the American sections of Prosopis L. (Leguminosae: Mimosoidae). Bull of the International Group for the study of Mimososidae 20:71-83.

Burkart A (1937) Una nueva especie de Prosopis del Monte occidental argentino. Revista Argentina de Agronomía 4:39-42.

Burkart A (1976) A monograph of the genus Prosopis (Leguminosae subfam. Mimosoidae). Journal Arnold Arboretum 57:219-525.

Carman NJ (1973) Systematic and ecological investigations in the genus Prosopis (Mimosaeae) emphasizing the natural products chemistry. PhD. Thesis, University of Texas, Austin, USA.

Garrigan D and Hedrick PW (2003). Perspective: Detecting adaptive molecular polymorphism: lessons from the MHC. Evolution 57:1707-1722.

Hunziker JH, Naranjo CA, Palacios RA, Poggio L and Saidman BO (1986) Studies on the taxonomy, genetic variation and biochemistry of Argentine species of Prosopis. Forest Ecology and Management 16:301-315.

Klein J (1980) Generation of diversity at MHC loci: Implications for T-cell receptor repertoires. In: Fougereau $\mathrm{M}$ and Dausset J (eds) Immunology 80, Academic Press, London, pp 239253.
Nei M (1978) Estimation of average heterozygosity and genetic distance from a small numbers of individuals. Genetics 89:583-590.

Neff JL, Simpson BB and Moldenke AR (1977) Flowers-flower visitor system. In: Orians GH and Solbrig OT (eds) Convergent Evolution in Warm Deserts. US/IBP Synthesis Series, Dowden Hutchinson and Ross, Stroudsburg, pp 204-223.

Palacios RA and Bravo ID (1981) Hibridación natural en Prosopis (Leguminosae) en la Región Chaqueña Argentina. Evidencias morfológicas y cromatográficas. Darwiniana 23:3-35.

Ramírez L, De La Vega A, Razkin N, Luna V and Harris PJC (1999) Analysis of the relationships between species of the genus Prosopis revealed by the use of molecular markers. Agronomie 19:31-43.

Roig FA (1993) Aportes a la etnobotánica del género Prosopis. In: Unidades de Botánica y Fisiología Vegetal, IADIZA (eds) Contribuciones Mendocinas a la Quinta Reunión Regional para América Latina y el Caribe de la Red de Forestación del CIID, pp 99-121.

Saidman BO (1985) Estudio de la variación alozímica en el género Prosopis. PhD Thesis, FCEyN, Universidad de Buenos Aires, Buenos Aires, Argentina.

Saidman BO and Vilardi JC (1987) Analysis of the genetic similarities among seven species of Prosopis (Leguminosae: Mimosoideae). Theoretical Applied Genetics 75:109-116.

Saidman BO and Vilardi JC (1993) Genetic variability and germplasm conservation in the genus Prosopis. In: Puri S (ed) Nursery Technology of Forest Tree Species of Arid and Semiarid Regions. Winrock-Oxford \& IBH Publishing Co. PVT. LTD., New Delhi, Bombay, Calcuta, pp 187-198.

Saidman BO, Vilardi JC, Pocoví MI and Acreche N (1996) Isozyme studies in Argentine species of the Section Strombocarpa, Genus Prosopis (Leguminosae). J Genetics 75:139-149.

Saidman BO, Vilardi JC, Montoya S and Poggio L (1997) Genetic variability and ploidy level in species of Prosopis (Leguminosae). Bol Soc Argent Bot 32:217-225.

Saidman BO, Bessega C, Ferreyra LI and Vilardi JC (1998)a Random amplified polymorphic DNA (RAPDS) variation in hybrid swarms and pure populations of genus Prosopis. In: Bruns S, Mantell S, Tragärdh C and Viana AV (eds) Recent Advances in Biotechnology for Tree Conservation and Management International Foundation for Sciences. Stockholm, pp 122-134.

Saidman BO, Vilardi JC, Montoya S, Dieguez MJ and Hopp HE (1998)b Molecular markers: A tool for the understanding of the relationships among species of Prosopis (Leguminosae, Mimosoidae). In: Puri S (ed) Tree Improvement: Applied Research and Technology Transfer. Science Publishers Inc. USA, pp 311-324.

Saidman BO, Bessega C, Ferreyra LI, Julio N and Vilardi JC (2000) The use of genetic markers to assess population structure and relationships among species of genus Prosopis (Leguminosae). Bol Soc Argent Bot 35:315-324.

Simpson BB (1977) Breeding system of dominant perennial plants of two disjunct warm desert ecosystems. Oecologia 27:203-226.

Simpson BB, Neff JL and Moldenke AR (1977) Prosopis flowers as a resource. In: Simpson BB (ed) Mesquite: Its Biology in Two Desert Ecosystems, US/IBP Synthesis Series, Dowden Hutchinson and Ross, Stroudsburg, pp 84-107. 
Solbrig OT and Bawa KS (1975) Isoenzyme variation in species of Prosopis (Leguminosae). Journal Arnold Arboretum 56:398-412.

Solbrig OT and Cantilo PD (1975) Reproductive adaptations in Prosopis (Leguminosae, Mimosoisae). Journal Arnold Arboretum 56:185-210.

StatSoft, Inc (1995) STATISTICA for Windows [Computer program manual]. Tulsa, OK: StatSoft, Inc., 2300 East 14th Street, Tulsa, OK, http://www.statsoftinc.com.

Swofford DL and Selander RB (1981) BIOSYS-1.7: A FORTRAN program for the comprehensive analysis of electrophoretic data in populations genetics and systematics. J Hered 72:281-283.
Verga AR (1995) Genetische untersuchungen an Prosopis chilensis und Prosopis flexuosa (Mimosaceae) im trockenen Chaco Argentiniens. PhD Thesis, Universität Göttingen.

Vilardi JC, Saidman BO and Palacios RA (1988) Muestreo según variabilidad. In: Prosopis en Argentina. Documento preliminar elaborado para el I Taller Internacional sobre Recursos Genéticos y Conservación de Germoplasma en Prosopis. FCA. UNC-FAO, PIRB, pp 119-124.

Wright S (1951) The genetical structure of populations. Annals eugenics 15:323-354.

Associate Editor: Márcio de Castro Silva Filho 